\title{
Comparison of preprocessing techniques for coin recognition using image processing methods
}

\author{
T. Hemapriya ${ }^{1 *}$, K.S. Archana ${ }^{2}$, T. Anupriya ${ }^{3}$ \\ ${ }^{I}$ Department of Computer Science \& Engineering, Vels Institute of Science, Technology \& Advanced Studies(VISTAS), Chennai, India. \\ ${ }^{2}$ Department of Computer Science \& Engineering, Vels Institute of Science, Technology \& Advanced Studies(VISTAS), Chennai, India. \\ ${ }^{3}$ Department of Computer Science \& Engineering, Vels Institute of Science, Technology \& Advanced Studies(VISTAS), Chennai, India. \\ *Corresponding author E-mail:hemapriyat7@gmail.com
}

\begin{abstract}
Coin is very important role in human's day life. For daily routine like shop, super market, banks etc the coins to be used. The coin is important part of economies and currency and it is used to pay for goods and also for our needs. Here the Indian coin has many number of count five rupee, ten rupee, two rupee, from this any one of the coin we are going to extract the texture feature for our Indian coin, first step is to preprocess the image is that method to enhance the image and remove the noise from enhanced image. For extracting clear information the image has to be preprocessed through some of the filtering techniques such as image size has to be resized, changing the contrast of the image, changing RGB to grayscale conversion for further operation such as segmentation and classification. At last the values to be compared by using PSNR, SNR, MSE of Filter noise removal with respective coin images.
\end{abstract}

\section{Keywords: Image preprocessing, filtering, gaussian filter, mean filter, median filter, wienner filter}

\section{Introduction}

In daily routine the coin draws a small part of real life such as shopping, purchase a small goods from shops, banks etc.. The coin classification system recognizer, the given coin has the certain measurement of the coin image. The measurement of the coin has physical properties like the weight, radius and conductivity using image processing, the feature has the important role in area of image processing. Then the feature extraction to get the clear feature of the image by using classification and recognition of image. It is able to extract the feature of obverse side and reverse side of the old Indian 5rupee coin and new Indian 5 rupee coin. The input image has to be classified by neural network. In this paper neural pattern is presented to recognized system, In this it can be rotate by any number of degree. Then only it can recognized what problem is affected in the coin image. In this algorithm classified the old Indian 5 rupee coin and new Indian 5 rupee coin, that the coin is recognized based on the closeness with one of the stored feature 1.size(radius), 2.color(color moments), 3.feature, 4.edge histogram.

\section{Literature survey}

Ashish khare[1] presented a method of pre-processing techniques for coin recognition using pattern recognition methods. The main aim of this paper was to get clear information, first the image has been converted to RGB color to grey color. Next, to extract useful information the image again converted to noised process using improved techniques.

Abdalla Mohamed Hambal, Dr. Zhijun Pei , Faustini Libent Ishabailu [2] presented a Noise reduction method, the process starts to removing noise from the image, there are many types of noise in the image but here finding a noise in the coin and have to remove by using suitable filter to get clear coin to segment as a part to next level.
Anurag Verma [3] Presented a research work of using Gaussian filter through image processing. Gaussian filter plays a important role to enhance the image to extract detail information for noise removal by using graphical software. It is used to reduce the noise from the image and reduce the image details. It is used to modify and enhancing the image .It includes to smooth, sharp and enhance the coin image

Raajan.P, Muthuselvi.S, Agnes Saleema [4] proposed a method for removing unwanted noise using Wiener Filter linear motion in coin image. The image has to be compared by various technique such as MSE optimal with linear filter of images to clear the noise and blurring in the coin image. Wiener filter is a filter used to produce the random process by linear time-invariant (LTI) filtering of an observed that noise is removed, that it has the stationary signal. According to author, to process the image first the image has to be enhanced through frequency domain image restoration method for coin recognition to find clear information.

Suman Shrestha[5] presented a method for coin pattern recognition using the filtering technique linear Gaussian for removing noise in image. For recognition the coin matching first the image has to be enhanced through some of the filtering technique such as median filter, Gaussian filter etc. to remove noise the image has to be smoothing first. At last the patched are identified through signal for find edges, but sometime it will affect the boundar.

Harsh Prateek Singh[6] presented the Mean filtering is most commonly used for reducing noise in the coin image. The mean filtering with a $3 \times 3$ kernel. According to author, salt and pepper noise used for It shows an image containing 'salt and pepper noise. It shows the effect of smoothing noisy of coin image with a $3 \times 3$ mean filter. The noise pixel values are very different from the surrounding values; they will calculate the pixel average by the mean filter. 


\section{Materials and methods}

\section{Capture image}

For recognizing the image, the picture has to be captured using digital camera with some highest pixel resolution. For extracting useful information the pixel resolution should to 2 Mega Pixel resolution of digital camera to get best results. so image processing plays a vital role for preprocessing method shown in Fig. 1.

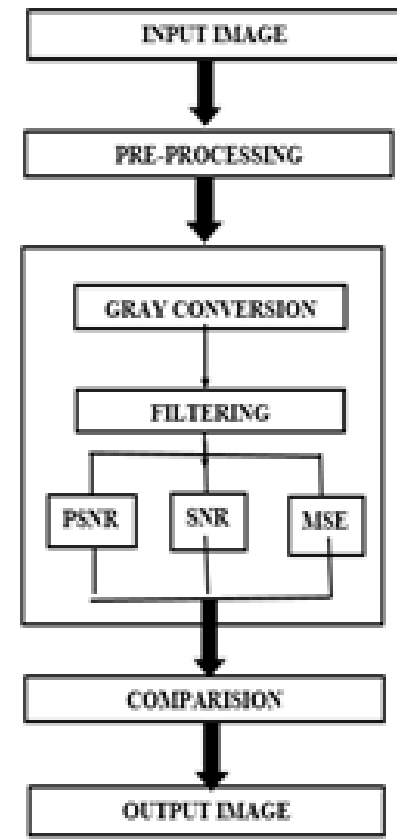

Fig. 1: Structure of preprocessing methods

\section{Image processing}

The main aim of this pre-processing is to improve the data on unwanted noise for further processing. For obtaining successive result the preprocessing method is initial for image analyzing. Most problems occur due to blurred image and noised image. The operation has to be drawn through calculating new pixel from neighborhood pixel. Next, the image has to be resized, finally gray scale image have to be proposed for analyzing the picture from RGB to grayscale conversion.

\section{Gaussian filter}

In image preprocessing the gaussian filter plays a important role to remove unwanted noise. It is used for image recognizing for blurring image. This effect is worn in the graphics software .It is used to trim down the noise from the image and reduce the image details. It is used to modify and enhancing the image .It includes to smooth, sharp and enhance the coin image. At last Comparison of PSNR, SNR, MSE of Gaussian Filter noise removal with respective coin image shown in Fig 3.

$$
G(x)=\frac{1}{\sqrt{2 \pi \sigma^{2}}} e^{-\frac{x^{2}}{2 \sigma^{2}}}
$$

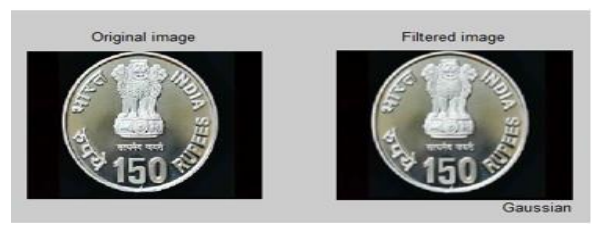

Gaussian filter

\begin{tabular}{|r|r|r|r|}
\hline Coin No & PSNR & SNR & MSE \\
\hline 1 & 24.33 & 13.94 & 49.83 \\
\hline 2 & 23.29 & 13.52 & 48.27 \\
\hline 3 & 23.29 & 12.6 & 59.78 \\
\hline 4 & 26.5 & 9.89 & 81.66 \\
\hline 5 & 23.28 & 14.22 & 42.2 \\
\hline 6 & 24.64 & 10.52 & 63.99 \\
\hline 7 & 23.48 & 13.97 & 41.26 \\
\hline 8 & 19.76 & 11.63 & 66.82 \\
\hline 9 & 27.3 & 9.77 & 82.83 \\
\hline 10 & 34.15 & 12.59 & 59.79 \\
\hline
\end{tabular}

(A) GAUSSIAN FILTER VALUES

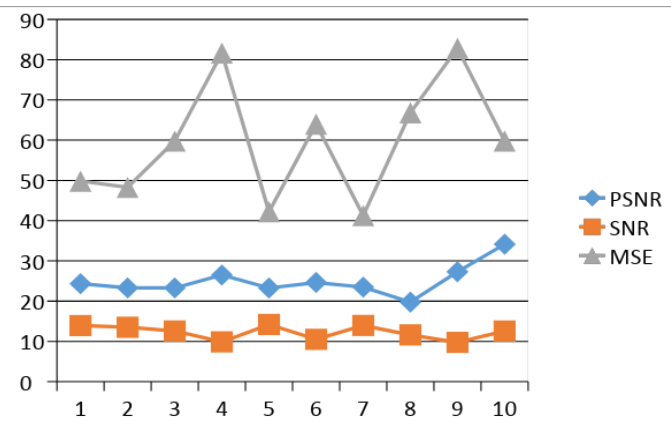

Fig. 2: Comparison of PSNR, SNR, MSE of gaussian filter noise removal with respective images

\section{Wiener filter}

The filter used to make estimated targeted region through noisy process. Sometimes the pixel value has to be corrupted due to unknown signal value. In this paper the input image and noised image has to be compared by the value of PSNR, SNR and MSE of Wiener Filter noise removal with respective coin images. The cross correlation between the signal is identified through image wiener method. To estimate the signal the continuous time monitoring has to compare for final classification value. At last to extract valuable pixel the images has to be enhanced. According to auto correlations the final pixel is valuated to remove denoised image shown in Fig 3.

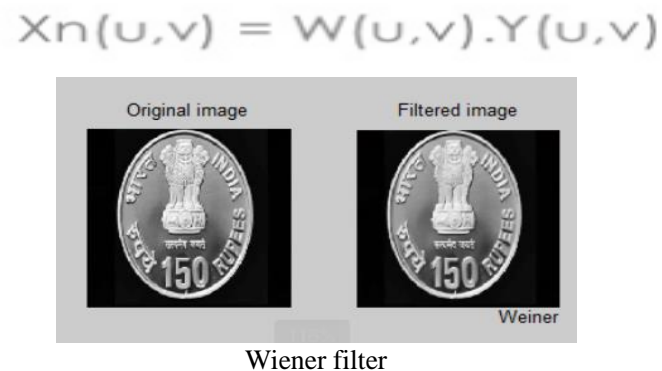

\begin{tabular}{|r|r|r|r|} 
Coin N & PSNR & SNR & MSE \\
\hline 1 & 30.26 & 13.05 & 40.48 \\
\hline 2 & 29.65 & 13.99 & 39.12 \\
\hline 3 & 30.4 & 12.86 & 57.99 \\
\hline 4 & 27.53 & 10.14 & 79.33 \\
\hline 5 & 30.54 & 13.24 & 38.53 \\
\hline 6 & 31.24 & 10.13 & 63.37 \\
\hline 7 & 32.81 & 13.12 & 41.28 \\
\hline 8 & 27.13 & 13.02 & 56.89 \\
\hline 9 & 32.63 & 9.73 & 83.17 \\
\hline 10 & 32.58 & 12.97 & 57.27 \\
\hline
\end{tabular}

(A) WIENER FILTER VALUES 


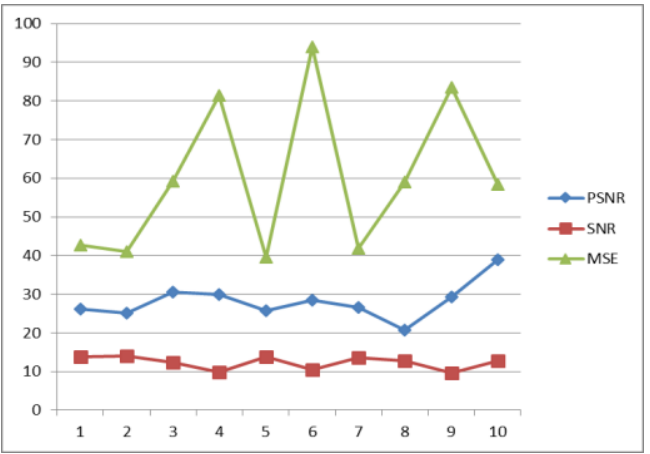

Fig. 3: Comparison of PSNR, SNR, MSE of wiener filter noise removal with respective images

\section{Median filter}

The median filter is a nonlinear filter technique, the noise is removed from the coin image by using the median filter The noise reduction has the pre-processing(for example: detecting the edge of the coin image). The median filter is used I digital image processing, while removing the noise we can see the clear image of the edges. It is used to smoothing the coin image, it will remove 'salt and pepper 'noise easily other than any noise. At last the comparison of PSNR, SNR, MSE of median filter noise removal with respective coin image shown in Fig 4.
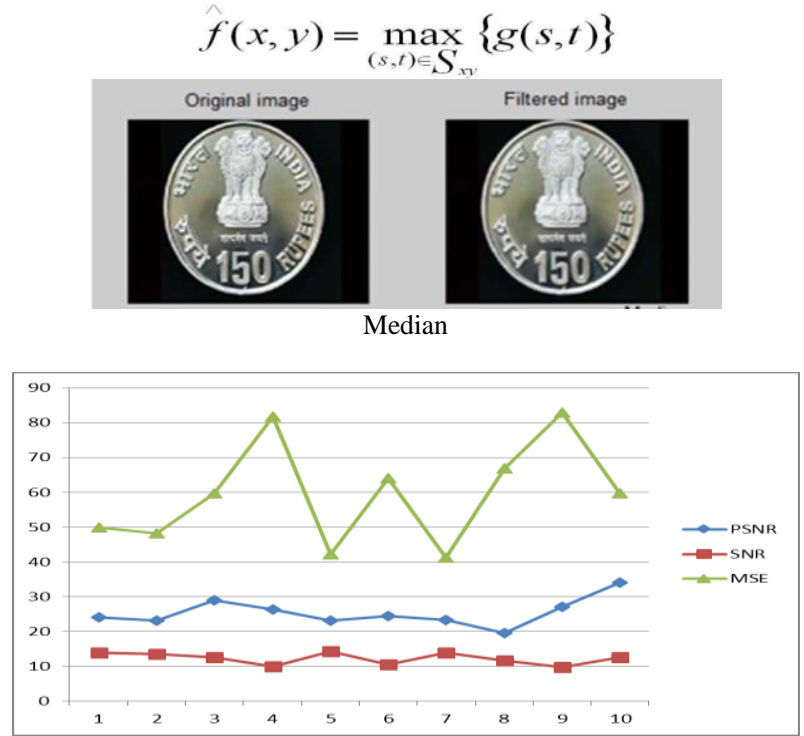

Fig. 4: Comparison of PSNR, SNR, MSE of median filter noise remova with respective images

\section{Mean filter}

Mean filter is most commonly used for reducing noise in the coin image. The mean filtering with a $3 \times 3$ kernel. It shows the $3 \times 3$ mean filters to smoothing noisy of coin image. The noise pixel values is differ from surrounding values, It will calculate the pixel average by the mean filter .It comparison of PSNR, SNR,MSE of mean filter noise removal with respective image shown in Fig 5.

$$
\hat{f}(x, y)=\frac{1}{m n} \sum_{(s, t) \in S_{x y}} g(s, t)
$$

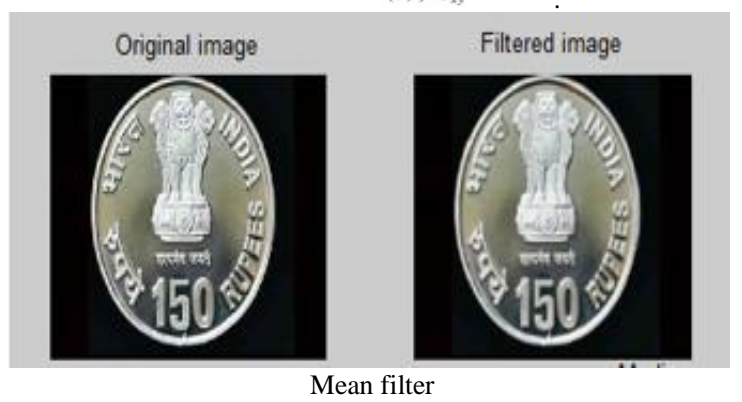

\begin{tabular}{|r|r|r|r|} 
COIN NO & PSNR & SNR & MSE \\
\hline 1 & 24.19 & 13.94 & 49.83 \\
\hline 2 & 23.15 & 13.52 & 48.27 \\
\hline 3 & 29.08 & 12.59 & 59.78 \\
\hline 4 & 26.33 & 9.89 & 81.66 \\
\hline 5 & 23.13 & 14.22 & 42.2 \\
\hline 6 & 24.49 & 10.53 & 63.99 \\
\hline 7 & 23.32 & 13.97 & 41.26 \\
\hline 8 & 19.63 & 11.63 & 66.82 \\
\hline 9 & 27.16 & 9.77 & 82.83 \\
\hline 10 & 34 & 12.59 & 59.79 \\
\hline
\end{tabular}

(C) MEAN FILTER VALUES

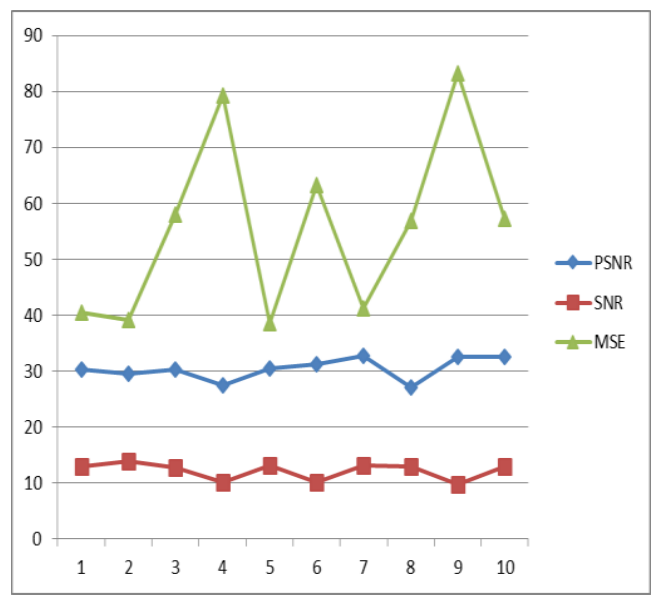

Fig. 5: Comparison of PSNR, SNR, MSE of mean filter noise removal with respective images

\section{Conclusion}

The coin is important part of economies and currency and it is used to pay for goods and also for our needs. Here the Indian coin has many number of count five rupee, ten rupee, two rupee, from this any one of the coin we are going to extract the texture feature for our Indian coin, first step is to preprocess the image is that method to enhance the image and remove the noise from enhanced image. To preprocess the image first image to be captured through digital camera but some of the images are noised and blurred. So image to be enhance some of the preprocessing methods are used. In this work, MATLAB tool used to resize image, adjusting the contrast level and finally four preprocessing techniques compared for further analyzing. At last through comparison the better result is obtained by wiener filter.

\section{References}

[1] Bawa S \& Modi S, "India Automated Coin Recognition System using ANN", International Journal of Computer Applications, Vol.26, No.4, (2011).

[2] Hassoubah RS, Aljebry AF \& Elrefaei LA, "Saudi Riyal Coin Detection and Recognition", IEEE Second International Conference On Image Information Processing, (2013).

[3] Velu CM, Vivekanadan P \& Kashwan K, "Indian Coin Recognition and Sum Counting System of Image DataMining Using Artificial Neural Networks", International Journal of Advanced Science and Technology, Vol.31, (2011).

[4] Unnikrishnan G \& Sajith sethu P, "Automatic Coin Recognition Using Local", International Journal of Computer Applications, (2006).

[5] Malik S, Bajaj P \& Kaur M, "Sample Coin Recognition System using Artificial Neural Network on Static Image Dataset", International Journal of Advanced Research in Computer Science and Software Engineering, Vol.4, No.1, (2014).

[6] Velu CM, "Indian Coin Recognition System of Image Segmentation by Heuristic Approach and Hough Transform (HT)", Int. J. Open Problems Compt. Math., Vol.2, No.2, (2009). 
[7] Shelgikar N, "Indian Coin Recognition with Rotation Invariance using Radial Blur Technique", IEEE Second International Conference On Image Information Processing, (2014).

[8] Reisert M, Ronneberger O \& Burkhardt H, "An efficient gradient based registration technique for coin recognition", Proc. of the MUSCLE CIS Coin Competition Workshop, (2006).

[9] Quraishi MI, Das G, Dhal KG \& Das P, "Classification of Ancient Coin using Artificial Neural Network", International Journal of Computer Applications, (2013).

[10] Malik S, Bajaj P \& Kaur M, "Coin Recognition System using Artificial Neural Network on Static Image Dataset-A Review", International Journal of Advanced Research in Computer Science and Software Engineering, Vol.3, (2013). 